\title{
Small protein A and phospholipase D immunization serves a protective role in a mouse pneumonia model of Acinetobacter baumannii infection
}

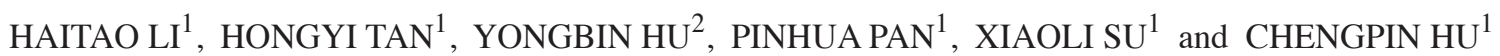 \\ ${ }^{1}$ Department of Pulmonary and Critical Care Medicine; ${ }^{2}$ Department of Pathological Medicine, \\ Xiangya Hospital, Central South University, Changsha, Hunan 410008, P.R. China
}

Received April 6, 2016; Accepted March 16, 2017

DOI: $10.3892 / \mathrm{mmr} .2017 .6688$

\begin{abstract}
Acinetobacter baumannii is an important pathogen that primarily causes hospital-acquired pneumonia. The present study sought to investigate whether small protein A (SmpA) and phospholipase D (PLD) are potential candidates for protective immunity against infection with A. baumannii. Mice immunized with the fusion proteins histidine (His)-SmpA and His-PLD exhibited a specific immunoglobulin $\mathrm{G}$ response. In a pneumonia model, active and passive immunization against SmpA and PLD protected mice from A. baumannii infection. The protection was demonstrated by a markedly improved survival rate, and reduced pulmonary bacterial load, infiltration and cytokine levels in the broncho-alveolar lavage fluid and the serum, although a combination of the two antigens did not provide improved protection compared with immunization with the individual antigens alone. In conclusion, it was identified that SmpA and PLD are highly immunogenic proteins, and potential antigen candidates for the development of effective vaccines or to prepare antisera to mitigate $A$. baumannii infection.
\end{abstract}

\section{Introduction}

Acinetobacter baumannii is a gram-negative, aerobic bacterium that causes a severe infection in immunocompromised patients (1), leading to bacteraemia, urinary tract infection, surgical site infection and, particularly, ventilator-associated pneumonia in intensive care patients (2). A. baumannii has emerged as an important pathogen due to its capacity to spread in a nosocomial environment and to display diverse mechanisms of antibiotic resistance (3). In recent years, epidemics

Correspondence to: Dr Pinhua Pan, Department of Pulmonary and Critical Care Medicine, Xiangya Hospital, Central South University, 87 Xiangya Road, Kaifu, Changsha, Hunan 410008, P.R. China E-mail: pinhuapan668@126.com

Key words: small protein A, phospholipase D, protective role, Acinetobacter baumannii caused by multidrug-resistant strains of $A$. baumannii have been widely investigated and described $(3,4)$. The easy acquisition of multi- or pan-drug resistance by this organism to clinically available antimicrobial agents has led to serious therapeutic complications (5-8). Multi-locus sequence typing (MLST) results (sequence type 191 is the most commonly-used) have indicated a local epidemic of carbapenem-resistant A. baumannii $(9,10)$, resulting in fewer therapeutic choices being available to combat this infection. Novel approaches to prevent and treat multi- or pan-drug-resistant infections are required. Immunological strategies that may function via different mechanisms, compared with antibiotics, are emerging as a viable option and may be able to circumvent complex multidrug-resistant mechanisms.

Outer membrane lipoprotein A, a small protein A (SmpA) homologue in Pseudomonas aeruginosa, is considered to be important in the formation and maintenance of cell membrane structure and integrity, and is a virulence factor in Gram-negative bacilli (11-13). Reverse immunology has indicated that SmpA may be an appropriate vaccine candidate against A. baumannii infection (14), despite a lack of investigation of the function of SmpA in A. baumannii. An essential role in the process of transmission of pathogens via blood has been demonstrated for phospholipase D (PLD) $(15,16)$, and it has been implicated to be a virulence factor of A. baumannii (16). Therefore, based on in silico analysis, PLD may be an appropriate subunit vaccine candidate (17).

The present study focused on the effects of SmpA and PLD immunization in a pneumonia model of $A$. baumannii infection using active and passive approaches. The present study additionally investigated whether the efficiency of combined immunization with both subunits has an advantage over separate immunizations. The results of the present study may facilitate an evaluation of the protection produced by immunological approaches against infection by drug-resistant A. baumannii, and lead to increased understanding of A. baumannii infection and immunization.

\section{Materials and methods}

Ethics statement. All animal procedures and experimental protocols were established according to the guidelines of 
the National Institutes of Health (Bethesda, MA, USA) and were approved by the Ethics Committee of Xiangya Hospital, Central South University (Changsha, China).

Bacterial strains and mice. A. baumannii strain ATCC 17978 was obtained from the American Type Culture Collection (Manassas, VA, USA). A. baumannii clinical isolates were previously obtained from respiratory intensive care unit patients in Xiangya Hospital, Central South University (Changsha, China) (18). In a previous study (18), DNA sequencing analysis using MLST demonstrated that the most frequently detected sequence type (ST) is ST191, followed by ST208 and ST425; these are the predominant sequence types in the carbapenem-resistant $A$. baumannii clinical isolates used in the present study.

A total of 128 female BALB/c mice (4-6 weeks of age, 14-16 g) were obtained and raised at the Department of Laboratory Animal Science, Central South University (Changsha, China) under specific pathogen-free conditions. Mice were maintained in a 12 -h light/dark cycle at $20-25^{\circ} \mathrm{C}$, and had free access to food and water. Previous clinical data have indicated that diabetes is a risk factor for the acquisition of A. baumannii infection and may lead to worse outcomes (19-21). All mice in the present study were intraperitoneally injected with a low dose $(70 \mathrm{mg} / \mathrm{kg}$ ) of streptozotocin (STZ) for 5 consecutive days, as repeated administration of STZ has been demonstrated to establish a diabetic model with long-term stability (22). After 1 week, random weekly blood glucose monitoring of mice that had received STZ indicated that glucose levels were $>16.7 \mathrm{mmol} / \mathrm{l}$, and that this was maintained for 12 weeks. The mice use in the present study were all diabetic models.

SmpA and PLD production. Using genomic DNA extracted from A. baumannii ATCC 17978, the SmpA and PLD genes were obtained via polymerase chain reaction (PCR) amplification, with sequences from gene accession number ABAYE2921 (SmpA) and A1S_2989 (PLD) serving as references. SmpA was amplified with the forward and reverse primers 5'-ATGCAA AAACTCGTGCTGA-3' and 5'-TTATAGTGGTGGGGC AGTTAAA-3', respectively; and PLD was amplified with the forward and reverse primers 5'-GACACCATATGGTTGACA TCCCTCAGGG-3' and 5'-GTGTCCTCGAGTTACATAAAG CCTTCCA-3', respectively. The PCR was performed under the following conditions: $94^{\circ} \mathrm{C}$ for $30 \mathrm{sec}, 56^{\circ} \mathrm{C}$ for $45 \mathrm{sec}$ and $72^{\circ} \mathrm{C}$ for $45 \mathrm{sec}$, for a total of 30 cycles. The amplified fragment was sequenced with assistance from Songong BioTech (Shanghai, China; data not presented) and subsequently cloned into the plasmid pThioHisA (Invitrogen; Thermo Fisher Scientific, Inc., Waltham, MA, USA) between the NdeI and XhoI restriction sites. The newly constructed plasmid was transformed into Escherichia coli BL21 (DE3) cells (Songong BioTech) with pre-cooling in $0.1 \mathrm{mM}$ calcium chloride solution, and the cells were grown in lysogeny broth medium (Qingdao Hope Biol-Technology, Co., Ltd., Qingdao, China) at $37^{\circ} \mathrm{C}$ for $8 \mathrm{~h}$. Subsequently, $0.2 \mathrm{mM}$ isopropyl b-D-1-thiogalactopyranoside was added to the culture medium to promote recombinant protein overexpression at $37^{\circ} \mathrm{C}$ for $4 \mathrm{~h}$. Following sonication of bacterial cells, the precipitate with inclusion bodies was collected by centrifugation at $12,000 \mathrm{x}$ g for $20 \mathrm{~min}$ at $4^{\circ} \mathrm{C}$. The precipitate with inclusion bodies was resuspended and dissolved in solubilisation buffer $(20 \mathrm{mM}$ Tris- $\mathrm{HCl} \mathrm{pH} 8.0$, $0.5 \mathrm{M} \mathrm{NaCl}, 8 \mathrm{M}$ urea). Following centrifugation at 12,000 x g for $20 \mathrm{~min}$ at $4^{\circ} \mathrm{C}$, the supernatant containing dissolved inclusion bodies was reserved for purification. The expressed recombinant proteins contained a six histidine (His)-tag, and were purified by refolding (completed when the concentration of urea decreased to $2 \mathrm{M}$ ) in vitro, followed by His-Ni affinity chromatography on HiTrap Q FF and HisTrap FF columns (both from GE Healthcare Life Sciences, Chalfont, UK), according to the manufacturer's protocol.

Immunoblotting. Subsequent to Ni-chelating affinity chromatography, the concentrations of the purified recombinant proteins were measured using a bicinchoninic acid Protein Assay kit (Beyotime Institute of Biotechnology, Shanghai, China), according to the manufacturer's protocol. Proteins $(20 \mu \mathrm{g})$ were isolated on 12\% SDS-PAGE gels, and the proteins were transferred to a polyvinylidene fluoride (PVDF) membrane. Blots were blocked in 5\% skimmed milk solution at $37^{\circ} \mathrm{C}$ for $1 \mathrm{~h}$ and briefly washed in TBS-Tween (v/v 0.5\%). The membrane was incubated with a pooled anti-His tag antibody (sc-8036; Santa Cruz Biotechnology, Inc., Dallas, TX, USA) at a dilution of $1: 1,000$ at $37^{\circ} \mathrm{C}$ for $1 \mathrm{~h}$ and was subsequently incubated with a bovine horseradish peroxidase-conjugated anti-mouse immunoglobulin (Ig) G secondary antibody (sc-2371; Santa Cruz Biotechnology, Inc.) at a dilution of $1: 10,000$ at $37^{\circ} \mathrm{C}$ for $4 \mathrm{~h}$. The PVDF membrane was developed using an enhanced chemiluminescence reagent (Thermo Fisher Scientific, Inc.) and was exposed to X-ray film.

Mouse immunization and pneumonia model. In order to select the antigen dose, mice were immunized following the protocols presented in Fig. 1A. Female BALB/C mice were immunized subcutaneously with a dose gradient (5, 25 and $125 \mu \mathrm{g}$ ) of His-SmpA or His-PLD and a separate adjuvant ( $0.1 \mathrm{mg}$ aluminium hydroxide). The mice were immunized three times with the same dosage at 2-week intervals (Fig. 1A). The appropriate doses were selected according to the dose of antigen and the immune response, indicated by the serum IgG titre.

Subsequent animal experiments were performed using a repeated immunization scheme (Fig. 1B) for active immunization. The mice were immunized subcutaneously with $25 \mu \mathrm{g}$ His-SmpA or $5 \mu \mathrm{g}$ His-PLD, a combination of both proteins, or adjuvant alone. ELISA analysis was used to determine the specific IgG response. In order to establish a pneumonia model to study active immunization, the mice were subsequently infected via aerosolized bacterial cells (clinical isolate ST191) produced by a nebulizer in an inhalation chamber, as previously reported (23). The mice were placed in a hermetically sealed Plexiglas container and exposed to a bacterial aerosol produced by atomizing a $12 \mathrm{ml}$ suspension $\left(10^{11}\right.$ cells $\left./ \mathrm{ml}\right)$ using a nebulizer driven by compressed air at $0.2 \mathrm{MPa}$. The nebulizer was connected to the sidewall of the chamber, and the aerosol occupied the entire space for $1 \mathrm{~h}$. The entire process was performed in a negative pressure environment. To verify that the inoculum had been inhaled, three mice from each group were sacrificed immediately following the procedure, and their lungs were harvested and quantitatively cultured on blood agar plates. 
A

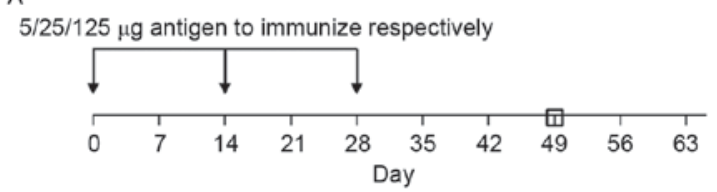

B

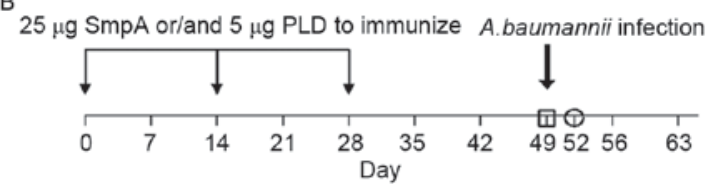

C

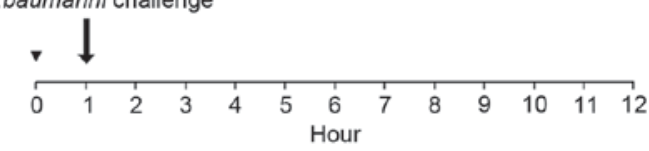

QSerum collected O Mice sacrificed

Figure 1. The protocols for (A) antigen dose selection, and (B) active and (C) passive immunization against Acinetobacter baumannii infection using a pneumonia model. SmpA, small protein A; PLD, phospholipase D.

For passive immunization, the mice were injected intravenously with $100 \mu \mathrm{l}$ antiserum taken from previous actively immunized mice $1 \mathrm{~h}$ prior to challenge with an intra-tracheal instillation of A. baumannii (Fig. 1C), as previously described (24). Tracheal cannulation using a needle was performed in an anaesthetised mouse, and $100 \mu \mathrm{l}$ of a bacterial suspension containing $10^{8}$ colony-forming units (CFU) was introduced. Control serum was taken from mice which received only an adjuvant treatment. The mice were observed for 15 days in order to determine the survival rate.

ELISA and cytokine determination. Serum samples were taken 21 days subsequent to the last immunization. Specific IgG antibody responses were measured by ELISA. Recombinant SmpA and PLD were used to coat microplates at $5 \mu \mathrm{g}$ total protein $/ 100 \mu \mathrm{l}$ in each well. Serum was added subsequent to blocking at $37^{\circ} \mathrm{C}$ for $30 \mathrm{~min}$ with bovine serum albumin (Hyclone; GE Healthcare Life Sciences, Logan, UT, USA), and the plates were incubated with bovine anti-mouse IgG secondary antibodies (Santa Cruz Biotechnology, Inc.; $1: 10,000)$ at $37^{\circ} \mathrm{C}$ for $1 \mathrm{~h}$ and developed with alkaline phosphatase. The endpoint titre was defined as the highest dilution at which the optical density at $405 \mathrm{~nm}$ was $\geq 0.1$ above that of the background; serum was replaced with PBS in the background wells $(25,26)$. Broncho-alveolar lavage fluid (BALF) and serum samples were collected at 3 days following aerosol exposure. Following anaesthesia, the left lung of each mouse was flushed with $1 \mathrm{ml}$ PBS three times, and the BALF was collected by retaining the wash. The serum sample was produced by centrifuging blood at $300 \mathrm{x} \mathrm{g}$, at $4^{\circ} \mathrm{C}$ for $10 \mathrm{~min}$, that had been harvested by cardiac puncture. The cytokine concentrations [interleukin (IL)-1 $\beta$, IL-6 and tumour necrosis factor- $\alpha$ (TNF- $\alpha)]$ in the BALF and serum samples were determined using the MILLIPLEX MAP Mouse TH17 Magnetic Bead Panel (Merck KGaA, Darmstadt, Germany) following the supplier's protocol.
Lung tissue bacterial burden and histology. The right upper lobe of each mouse lung was weighed and homogenized to produce a tissue suspension in $3 \mathrm{ml}$ PBS. The bacterial load in the lung homogenate was determined by plating 10 -fold dilutions on goat-blood agar plates. The plates were incubated at $37^{\circ} \mathrm{C}$ overnight, and the CFUs were counted. The right middle lobes of individual mouse lungs were fixed in $4 \%$ paraformaldehyde at $4{ }^{\circ} \mathrm{C}$ overnight and paraffin-embedded. The lung gland tissue sections $(3 \mu \mathrm{m})$ were stained with hematoxylin and eosin. A semi-quantitative analysis was performed by scoring from 0 (no lesion) to 4 (major and extended lesions) based on the following criteria: Vascular congestion, alveolar necrosis, neutrophil infiltration and macrophage infiltration (27).

Statistical analyses. Statistical analyses were performed using Prism software (version 6.05; GraphPad Software, Inc., La Jolla, CA, USA). Survival was compared using the non-parametric log-rank test. Student's t-test or one-way analysis of variance (ANOVA) followed by Tukey's multiple comparisons test were applied to assess differences in the IgG levels, CFU counts, histological scores and cytokine concentrations. $\mathrm{P}<0.05$ was considered to indicate a statistically significant difference. Data were expressed as the mean \pm the standard deviation.

\section{Results}

Immunization with SmpA and PLD produces a high IgG response to $A$. baumannii. The recombinant proteins His-SmpA and His-PLD, which exhibited molecular weights of $\sim 14.2$ and $\sim 45 \mathrm{kDa}$, respectively, were efficiently expressed. Proteins were purified to homogeneity and analysed by SDS-PAGE (Fig. 2). The purified proteins were used to immunize mice, and the antibody titres were calculated a week subsequent to the final immunization in order to determine an appropriate vaccine dose. Responses were efficiently elicited by His-SmpA immunization with a maximum titre of $1 \times 10^{4}$, whereas His-PLD immunization elicited a maximum titre of $1 \times 10^{3}$ (Fig. 3). The maximum antibody titres for SmpA occurred at vaccine dosages of 25 and $125 \mu \mathrm{g}$, whereas PLD titre did not vary with dosage. Dosages of 25 and $5 \mu \mathrm{g}$ were selected for vaccination with SmpA and PLD, respectively. Fig. 4 illustrates that no significant immune interference was noted in the combined immunization group (SmpA and PLD) for active immunization.

Immunization with SmpA and PLD reduces bacterial load in a pneumonia model. Nebulization of $1.0 \times 10^{11} / \mathrm{ml}$ bacterial cells delivered an inoculum in the range 2.0-3.0x $10^{6} \mathrm{CFU}$ to the mouse lungs. No mortalities had occurred in any group $72 \mathrm{~h}$ following the aerosol administration. Determination of the bacterial load in the lungs harvested from all mice demonstrated that SmpA and PLD active immunization therapy resulted in $\sim 2.0$-log decreases in the lung bacterial burden compared with the adjuvant-treated mice, and the combined immunization therapy resulted in no more than a $0.1-\log$ difference compared with the SmpA-only or PLD-only mice; however, the difference was significant (Fig. 5).

Immunization with SmpA and PLD alleviates pulmonary infiltration in a pneumonia model. Histopathologically, the 


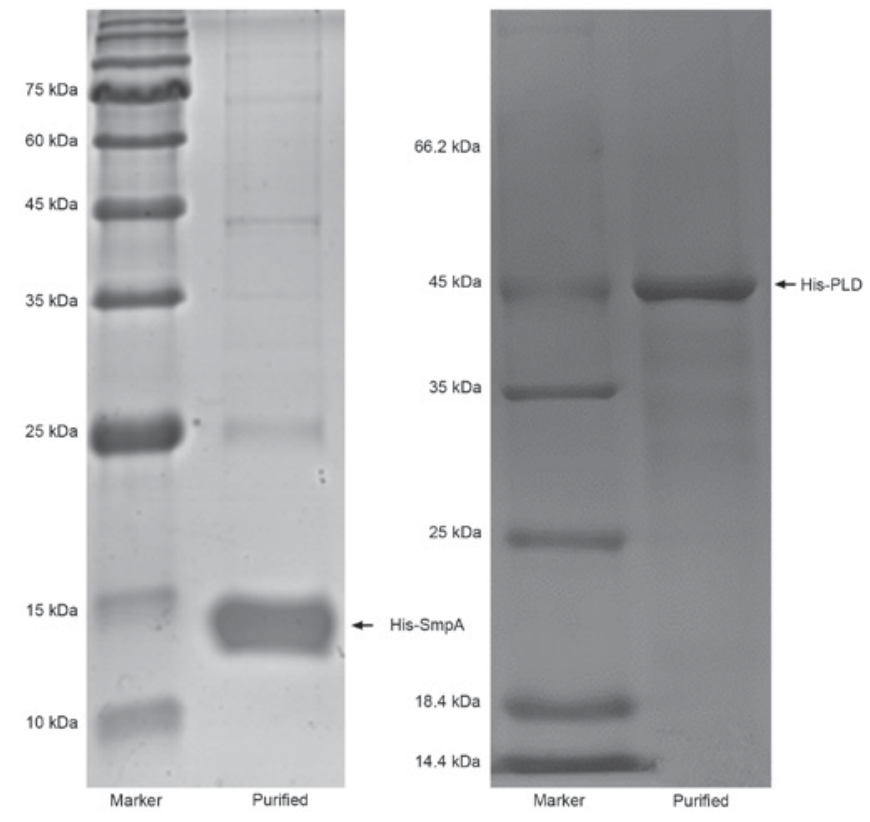

Figure 2. Expression and purification of the recombinant His-SmpA and His-PLD proteins on SDS-PAGE. SmpA, small protein A; PLD, phospholipase D.

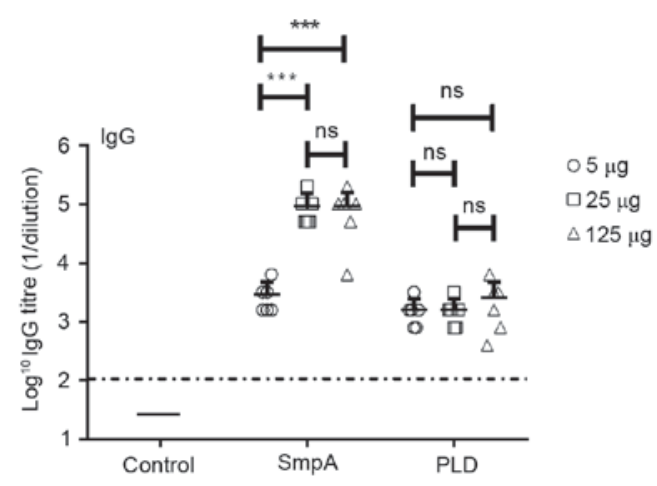

Figure 3. Antibody responses to immunization with SmpA and PLD. Serum samples were collected from SmpA-vaccinated, PLD-vaccinated and control mice when the immunization schedule was complete, and total antigen-specific IgG levels were measured by ELISA analysis $(\mathrm{n}=6$ mice/group). Data were analysed using a t-test. ${ }^{* * * *} \mathrm{P}<0.001$. ns, not significant; SmpA, small protein A; PLD, phospholipase D; IgG, immunoglobulin G.

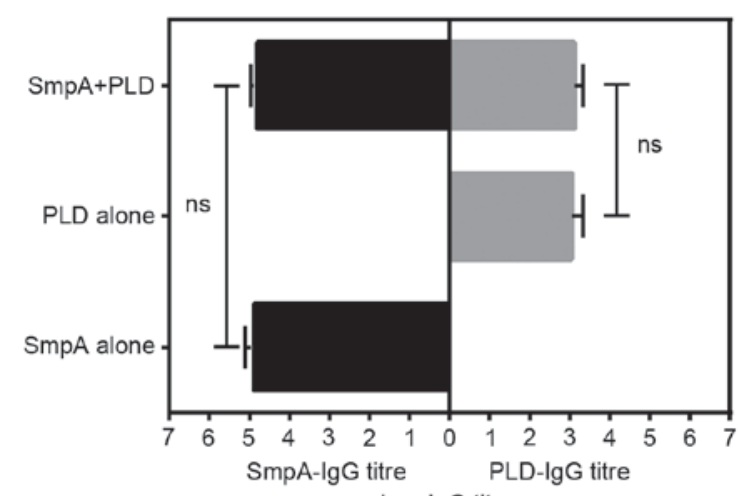

Log $\lg G$ titre

Figure 4. Specific IgG levels in serum induced by His-SmpA and His-PLD immunization were measured by ELISA following active immunization $(n=8$ mice/group). ns, not significant; SmpA, small protein A; PLD, phospholipase D; IgG, immunoglobulin G.

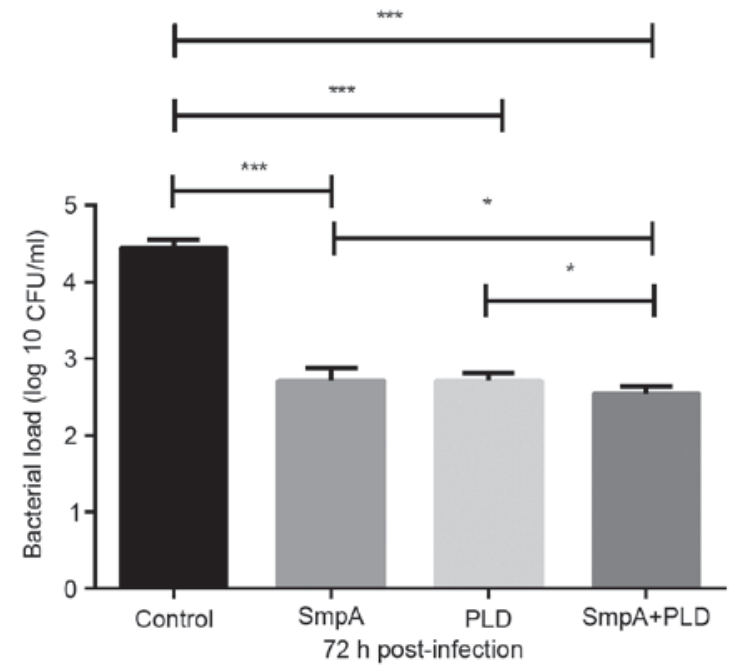

Figure 5. Effect of vaccination on lung tissue bacterial load. Mice were infected with strain ST191 $\left(10^{6} \mathrm{CFU}\right)$, and the lung bacterial load was determined $72 \mathrm{~h}$ post-infection ( $\mathrm{n}=8$ mice/group). Data were analysed using one-way analysis of variance, ${ }^{* * *} \mathrm{P}<0.001$, and $\mathrm{t}$-test, ${ }^{*} \mathrm{P}<0.05$. SmpA, small protein A; PLD, phospholipase $\mathrm{D}$; $\mathrm{CFU}$, colony forming units.

lungs from control mice $72 \mathrm{~h}$ subsequent to A. baumannii inhalation exhibited infiltrations of mixed neutrophils and lymphocytes in the perivascular and peribronchial spaces (Fig. 6A). By contrast, the histopathological alterations in the lungs of mice immunized with SmpA and PLD, which were sacrificed at $72 \mathrm{~h}$ post-infection, were milder and limited largely to the alveolar space, where small to moderate numbers of neutrophils and lymphocytes were observed (Fig. 6B-D). The histological scores of the lungs in each group were in accordance with the photomicrograph images of the lungs (Fig. 7). A significant difference between vaccinated groups and the control $(\mathrm{P}<0.001)$ was present; however, no differences between the vaccinated groups were observed by one-way ANOVA (control group, 7.05 \pm 0.17 ; SmpA group, 1.88 \pm 0.40 ; PLD group, 1.78 \pm 0.38 ; SmpA and PLD group, 1.58 \pm 0.40 ).

Immunization with SmpA and PLD influences the cytokine levels in BALF and serum in a pneumonia model. Sera and BALF were harvested simultaneously from each group of mice, and the IL-1 $\beta$, IL- 6 and TNF-a expression levels were determined (Fig. 8). The expression levels of the three cytokines were significantly reduced in all groups of vaccinated mice compared with control mice $(\mathrm{P}<0.001$; one-way ANOVA), Additionally, in the BALF, the TNF- $\alpha$ levels in mice immunized with SmpA and PLD were reduced compared with mice immunized with SmpA and PLD alone, as were IL-1 $\beta$ and IL-6 levels in the serum $(\mathrm{P}<0.05$; $\mathrm{t}$-test).

Passive immunization with SmpA and PLD increases mouse survival in a pneumonia model. In order to evaluate the protective efficacy of the antiserum, a pneumonia model was challenged via intra-tracheal instillation rather than aerosol inhalation, as the former is able to provide an increased concentration of A. baumannii, reaching a lethal dose. Treatment with antiserum improved the 15-day survival of infected mice compared with the control. In mice infected with ST191 (Fig. 9), a 50\% survival rate was observed with the anti-SmpA 

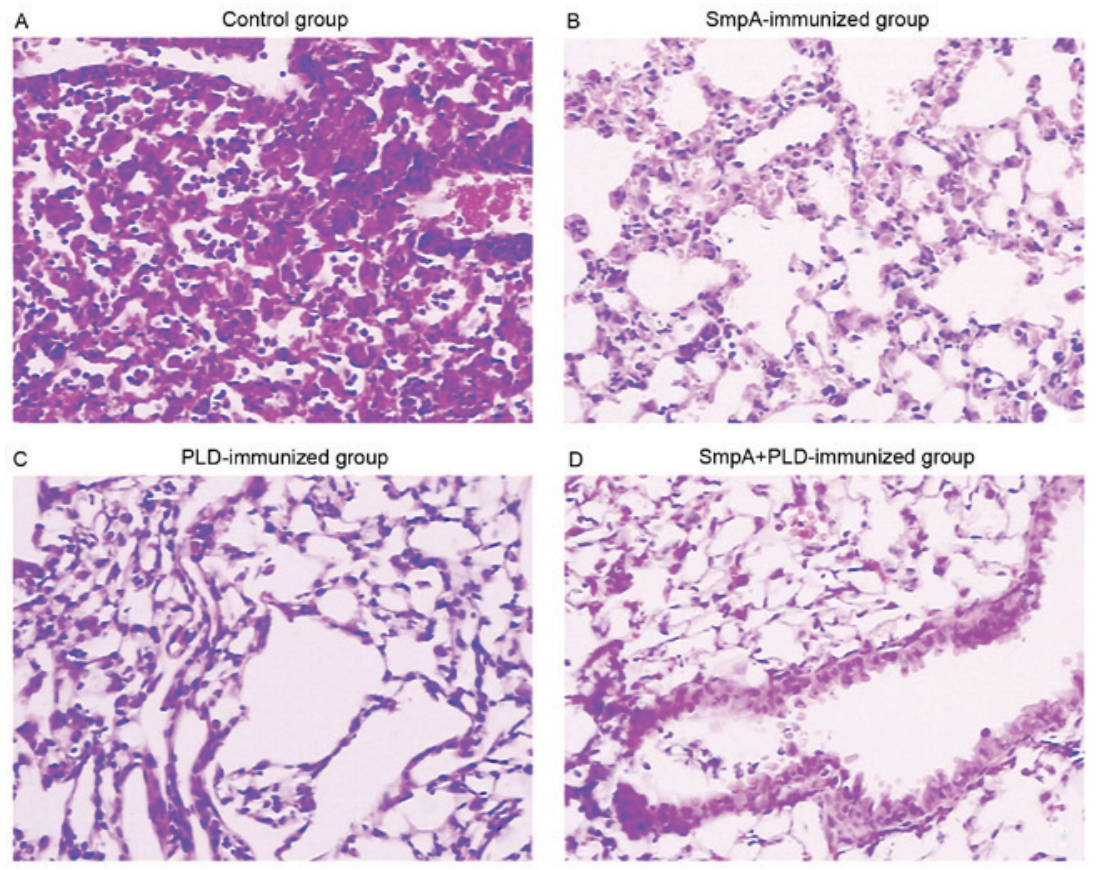

Figure 6. Photomicrograph images of lung histopathology. Photomicrograph images (magnification, x200; hematoxylin and eosin staining) of lung histopathology in (A) control mice, (B) SmpA-immunized mice, (C) PLD-immunized mice and (D) mice immunized with both SmpA and PLD. SmpA, small protein A; PLD, phospholipase D.

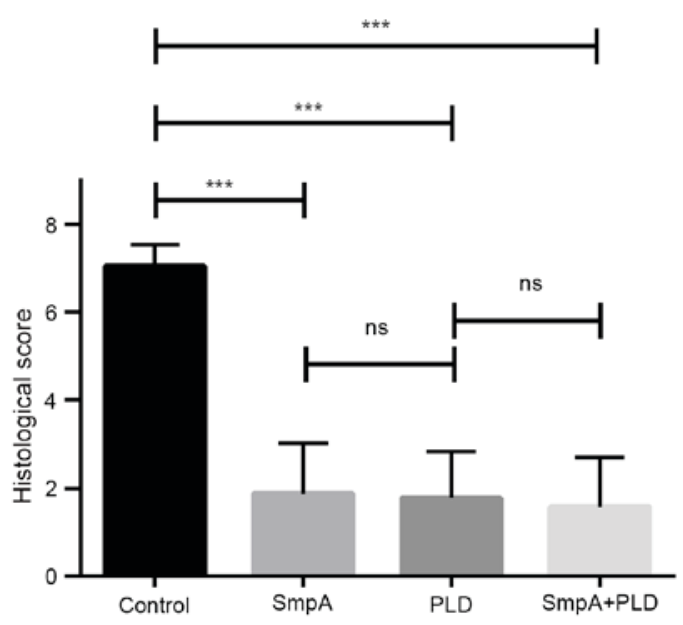

Figure 7. Effects of vaccination on histological scores. Data were analysed using one-way analysis of variance. ${ }^{* * *} \mathrm{P}<0.001$. ns, not significant; SmpA, small protein A; PLD, phospholipase D.

and anti-PLD sera alone, a $66.7 \%$ survival rate with combined immunization, and a $0 \%$ survival rate in the control group $(\mathrm{P}=0.0168$; log-rank test). In mice infected with ST208, a 50\% survival rate was observed with anti-SmpA serum, a $66.7 \%$ survival rate with anti-PLD serum, a $66.7 \%$ survival rate with combined immunization, and a $16.7 \%$ survival rate in the control group $(\mathrm{P}=0.0380$; log-rank test). In mice infected with ST425, a 33.3\% survival rate was observed with the anti-SmpA and anti-PLD sera alone, a 50\% survival rate with combined immunization, and a $0 \%$ survival rate in the control group $(\mathrm{P}=0.0162$; log-rank test). However, no significant differences were observed between the groups immunized with antisera containing a single component or a combination upon infection with any strain.

\section{Discussion}

During the past decade, A. baumannii has emerged as one of the principal antibiotic-resistant bacterial pathogens worldwide. The strategy of developing a vaccine against A. baumannii for use in humans was a novel approach to fighting the increased risk of infection caused by these multidrug-resistant strains.

A programme was undertaken to identify a series of candidate antigens for A. baumannii (28-34). Individual outer membrane proteins, including outer membrane protein A (OmpA) (29,31), biofilm-associated protein (Bap) (35), and Acinetobacter trimeric autotransporter (Ata) (31), have been identified as effective candidates for vaccines that immunologically intervene in A. baumannii infection. However, as the most promising candidate, OmpA did not reduce the bacterial load in mouse lung tissue $(30,32)$, and it was not certain that Bap was expressed in all strains (35). Antibodies against Ata have in vivo antibacterial activity; however, no evidence has demonstrated that Ata antibodies are sufficient to reduce post-infection mortality in an animal model (31). There are additional concerns regarding adaptation to immune pressure via downregulation of antigens, and purification processes may alter the conformation of an antigen (36). However, multicomponent vaccines, including inactivated whole cells (28), outer membrane complexes (8), and outer membrane vesicles of A. baumannii, have been demonstrated to be effective immunogens that have protected mice from bacterial challenge via active or passive immunization strategies (30). Compared with single vaccines, multicomponent vaccines may increase vaccine coverage; however, legitimate concerns exist about the difficulties involved in standardizing all components and the presence of impurities that may produce side effects $(8,28,30)$. Current vaccination strategies against A. baumannii have aimed at accentuating the strengths of 

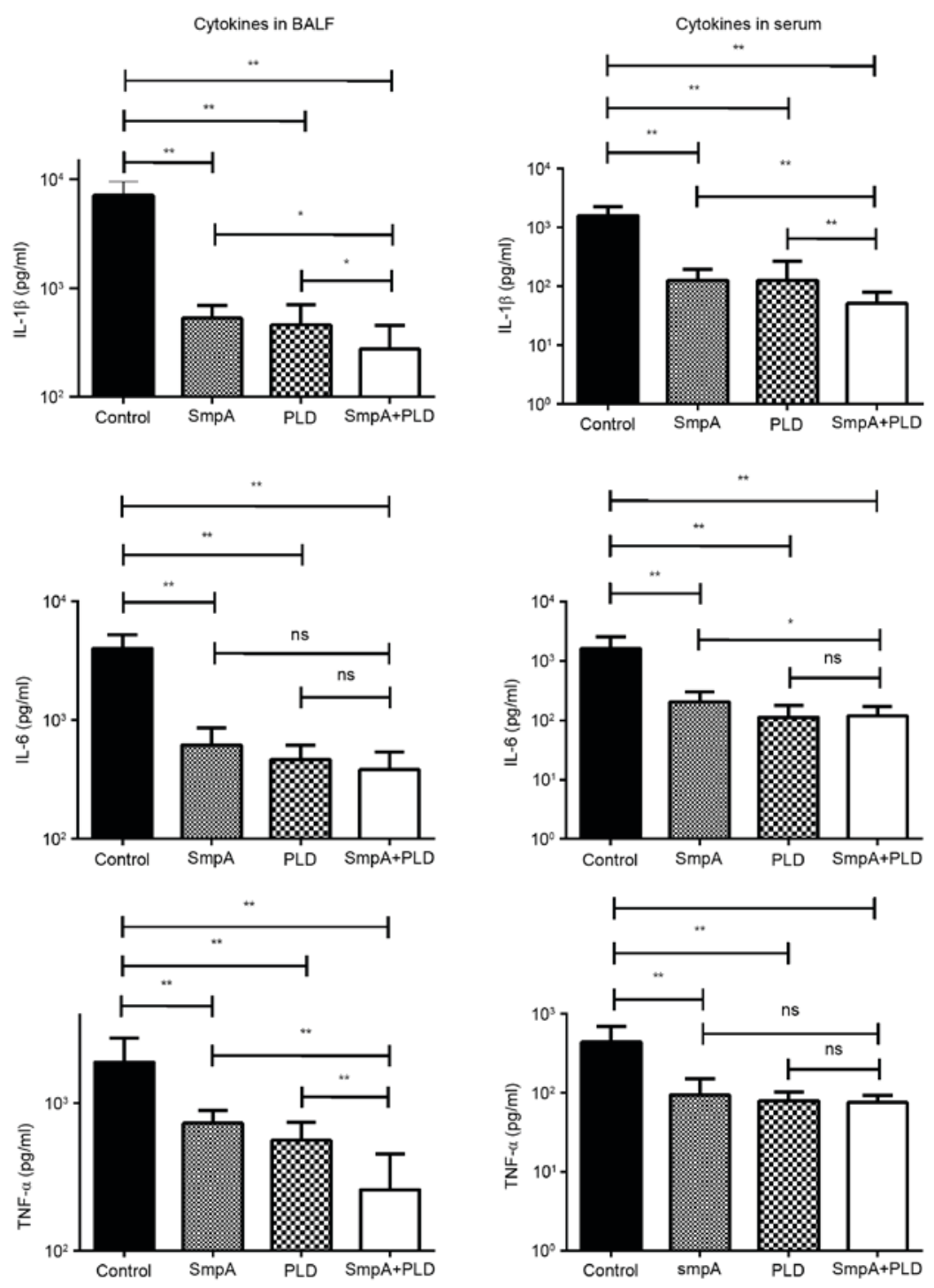

Figure 8. Effects of vaccination on pro-inflammatory cytokine levels post-infection. The levels of IL-1 $\beta$, TNF-a, and IL-6 in BALF and serum from vaccinated or control mice ( $\mathrm{n}=8$ mice/group) were determined. ${ }^{* *} \mathrm{P}<0.001,{ }^{*} \mathrm{P}<0.05$. BALF, broncho-alveolar lavage fluid; IL, interleukin; TNF, tumour necrosis factor; SmpA, small protein A; PLD, phospholipase D.
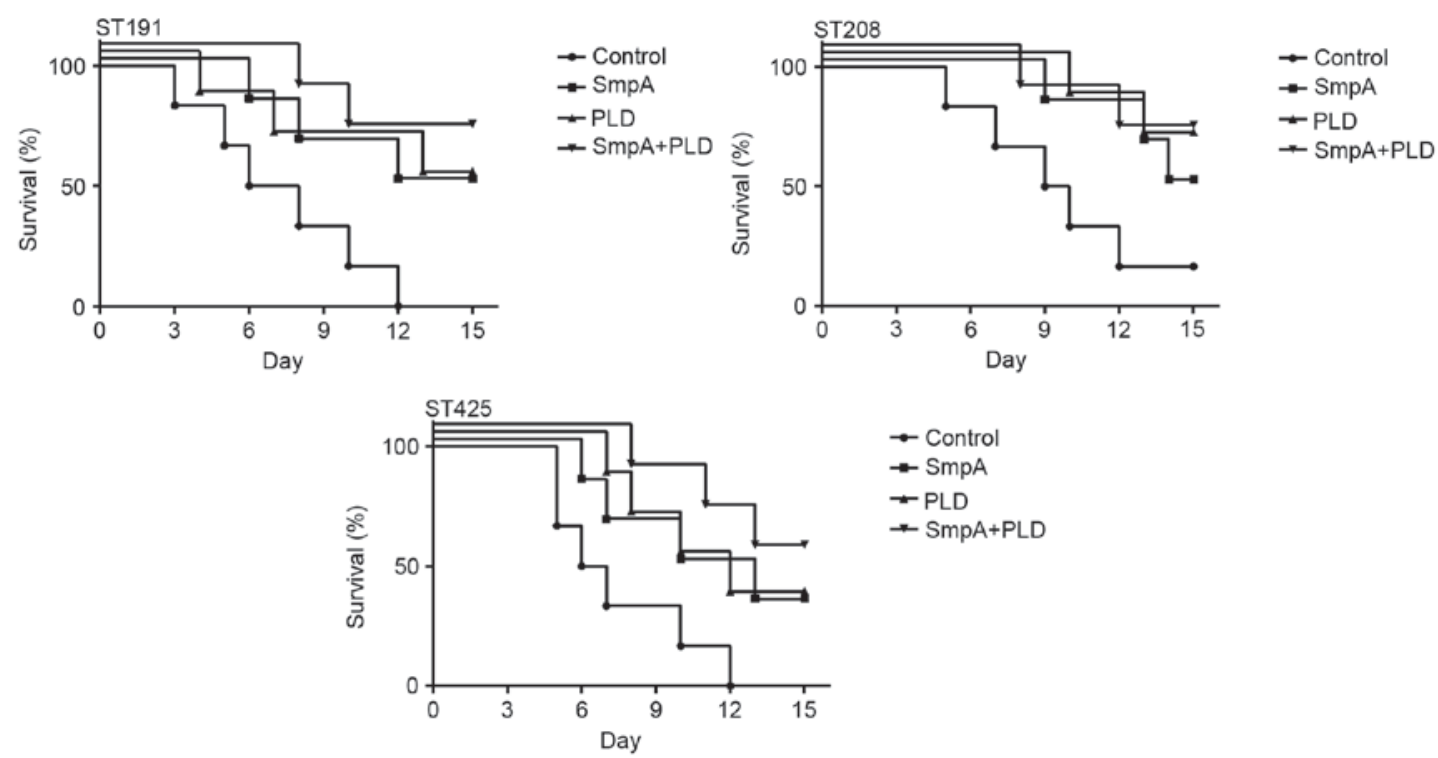

Figure 9. Effects of vaccination on survival in a mouse pneumonia model of Acinetobacter baumannii infection. Mice were monitored twice a day for 15 days ( $n=6$ mice/group). ST, sequence type; SmpA, small protein A; PLD, phospholipase D. 
single- and multi-component approaches rather than mitigating their weaknesses. Therefore, a vaccine consisting of a combination of several purified single components may constitute a new strategy. SmpA and PLD may be potential candidates for A. baumannii vaccines. Reverse vaccinology, and progress in genomics, proteomics and in silico technology, have allowed for the selection of promising theoretical targets $(22,26)$. The present study investigated whether two such proteins were efficient in practice and whether the proteins stimulated each another to enhance the potency of the immunization.

Recombinant SmpA and PLD were efficiently produced in E. coil cells. In order to facilitate the expression and refolding of SmpA and PLD as natural epitopes for the induction of specific IgGs, fusion proteins with His-tags at the $\mathrm{N}$ termini of SmpA and PLD were engineered and purified by affinity chromatography using a metal-chelating resin (37). The purification protocol ensured that the purified fusion proteins in in the present study were folded correctly and exhibited natural immunogenicity.

In the present study, active and passive immunization strategies were used. Active immunization elicited a persistent IgG response, and an appropriate vaccine dose was chosen to elicit the highest $\operatorname{IgG}$ titre for the minimum dosage. In the active immunization study, challenge with A. baumannii occurred at 3 weeks following the final immunization to allow for the loss of the innate immunity elicited by immunization with SmpA and PLD, thereby avoiding possible interference when assessing an adaptive immune response. The groups vaccinated with SmpA- and PLD-IgG exhibited a reduced bacterial load in the lung tissue, reduced lung infiltration and expression of mediating cytokines in the BALF and serum, which improved 15-day survival. As a result, the IgG titre of SmpA was increased compared with the PLD titre; however, the bacterial load in the lung in the PLD-only vaccine group was not significantly different compared with that in the SmpA-only group, indicating that the PLD content may be reduced compared with that of SmpA, although it may serve an important role in bacterial metabolic activity. The mice in the groups vaccinated with the combination of SmpA and PLD exhibited a decreased bacterial load in the lungs compared with mice vaccinated with the individual proteins; in addition, certain cytokine levels in the BALF or the serum were reduced. The cytokine levels in the BALF reflect lung tissue inflammation caused by infection with $A$. baumannii, while the cytokine levels in the serum reflect the systemic inflammatory responses. The results of the present study are consistent with the results of the analysis of pathological alterations in the lung tissue. However, pulmonary infiltration, as evaluated by the histological score, was not significant in groups immunized with SmpA, PLD or a combination of the two, and nor were survival rates. The results of the present study indicated that the bacterial load was not reduced sufficiently to confer a clinical advantage to the group immunized with the combined proteins. Additionally, the present study demonstrated that active and passive immunization with SmpA and PLD was efficient, and a combination appears to be unnecessary to protect against infection by a particular strain. It may be difficult for a recombinant protein vaccine to provide effective protection against different clinical strains. The three different strains selected to test the passive strategy demonstrated that SmpA and PLD are conserved in
A. baumannii and serve protective roles against $A$. baumannii pneumonia. The passive immunization protocol in the present study, performed on healthy animals with subsequent bacterial challenge, may not be optimal; however, it is sufficient to draw the conclusion that passive immunizations with SmpA and PLD antisera were efficient. In conclusion, active immunization may serve a clinical role in the prevention of infection in a high-risk population, whereas passive immunization may be applied to therapeutic treatment with antisera for infected individuals. It may be appropriate to discuss the association between the titre of the antiserum and survival rates in passive immunization; however, it has been traditionally hypothesized that an increased titer of antiserum results in a more potent antibacterial effect, without concern for adverse reactions caused by antiserum rejection. Future studies will investigate the therapeutic potency of the antisera of the proteins in the present study by applying them to clinical treatment.

Using an immunological approach with a pneumonia model for A. baumannii infection, and active and passive immunization strategies, the present study demonstrated that SmpA and PLD are important candidate antigens that effectively control infection with A. baumannii. Due to bacterial mutation, and to cover as many A. baumannii strains as possible, immunization with a mixture of subunits of SmpA and PLD may be required.

\section{Acknowledgements}

The present study was supported by the China Hunan Provincial Science and Technology Department (grant no. 2015SK20401).

\section{References}

1. Peleg AY, Seifert H and Paterson DL: Acinetobacter baumannii: Emergence of a successful pathogen. Clin Microbiol Rev 21: 538-582, 2008.

2. Zarrilli R, Casillo R, Di Popolo A, Tripodi MF, Bagattini M, Cuccurullo S, Crivaro V, Ragone E, Mattei A, Galdieri N, et al: Molecular epidemiology of a clonal outbreak of multidrug-resistant Acinetobacter baumannii in a university hospital in Italy. Clin Microbiol Infect 13: 481-489, 2007.

3. Munoz-Price LS and Weinstein RA: Acinetobacter infection. N Engl J Med 358: 1271-1281, 2008.

4. Dijkshoorn L, Nemec A and Seifert H: An increasing threat in hospitals: Multidrug-resistant Acinetobacter baumannii. Nat Rev Microbiol 5: 939-951, 2007.

5. Gordon NC and Wareham DW: Multidrug-resistant Acinetobacter baumannii: Mechanisms of virulence and resistance. Int J Antimicrob Agents 35: 219-226, 2010.

6. Alekshun MN and Levy SB: Molecular mechanisms of antibacterial multidrug resistance. Cell 128: 1037-1050, 2007.

7. Zavascki AP, Carvalhaes CG, Picão RC and Gales AC: Multidrug-resistant Pseudomonas aeruginosa and Acinetobacter baumannii: Resistance mechanisms and implications for therapy. Expert Rev Anti Infect Ther 8: 71-93, 2010.

8. McConnell MJ, Domínguez-Herrera J, Smani Y, Lopez-Rojas R, Docobo-Perez F and Pachon J: Vaccination with outer membrane complexes elicits rapid protective immunity to multidrug-resistant Acinetobacter baumannii. Infect Immun 79: 518-526, 2011.

9. Gurung M, Rho JS, Lee YC, Kim HS, Moon SY, Yu BH, Lee JH, Jang CW, Chung SY, Cho IH, et al: Emergence and spread of carbapenem-resistant Acinetobacter baumannii sequence type 191 in a Korean hospital. Infect Genet Evol 19: 219-222, 2013.

10. Ying J, Lu J, Zong L, Li A, Pan R, Cheng C, Li K, Chen L, Ying J, Tou H, et al: Molecular epidemiology and characterization of genotypic analysis of Acinetobacter baumannii strains isolates from south China. Jpn J Infect Dis 69: 180-185, 2016. 
11. Sklar JG, Wu T, Gronenberg LS, Malinverni JC, Kahne D and Silhavy TJ: Lipoprotein SmpA is a component of the YaeT complex that assembles outer membrane proteins in Escherichia coli. Proc Natl Acad Sci USA 104: 6400-6405, 2007.

12. Ryan KR, Taylor JA and Bowers LM: The BAM complex subunit BamE (SmpA) is required for membrane integrity, stalk growth and normal levels of outer membrane \{beta\}-barrel proteins in Caulobacter crescentus. Microbiology 156: 742-756, 2010.

13. Lewis C, Skovierova H, Rowley G, Rezuchova B, Homerova D, Stevenson A, Sherry A, Kormanec J and Roberts M: Small outer-membrane lipoprotein, SmpA, is regulated by sigmaE and has a role in cell envelope integrity and virulence of Salmonella enterica serovar Typhimurium. Microbiology 154: 979-988, 2008.

14. Moriel DG, Beatson SA, Wurpel DJ, Lipman J, Nimmo GR, Paterson DL and Schembri MA: Identification of novel vaccine candidates against multidrug-resistant Acinetobacter baumannii. PLoS One 8: e77631, 2013.

15. Batey RG: Pathogenesis of caseous lymphadenitis in sheep and goats. Aust Vet J 63: 269-272, 1986

16. Jacobs AC, Hood I, Boyd KL, Olson PD, Morrison JM, Carson S, Sayood K, Iwen PC, Skaar EP and Dunman PM: Inactivation of phospholipase D diminishes Acinetobacter baumannii pathogenesis. Infect Immun 78: 1952-1962, 2010.

17. Hosseingholi EZ, Rasooli I and Gargari SL: In silico analysis of Acinetobacter baumannii phospholipase $\mathrm{D}$ as a subunit vaccine candidate. Acta Biotheor 62: 455-478, 2014.

18. Tan H, Li ML, Wan Z, Pan PH, Su XL and Hu CP: Sequence Type 191 of Clonal Complex 92 was a Dominant Carbapenem-Resistant Acinetobacter baumannii that Causes Nosocomial Pneumonia in Intensive Care Units in a Teaching Hospital in Central China. Clinical Laboratory 62: 1421-1426.

19. Alsultan AA, Hamouda A, Evans BA and Amyes SG: Acinetobacter baumannii: Emergence of four strains with nove bla(OXA-51-like) genes in patients with diabetes mellitus. J Chemother 21: 290-295, 2009.

20. Furniss D, Gore S, Azadian B and Myers SR: Acinetobacter infection is associated with acquired glucose intolerance in burn patients. J Burn Care Rehabil 26: 405-408, 2005.

21. Metan G, Sariguzel F and Sumerkan B: Factors influencing survival in patients with multi-drug-resistant Acinetobacter bacteraemia. Eur J Intern Med 20: 540-544, 2009.

22. Nacci C, Tarquinio M, De Benedictis L, Mauro A, Zigrino A, Carratu MR, Quon MJ and Montagnani M: Endothelial dysfunction in mice with streptozotocin-induced type 1 diabetes is opposed by compensatory overexpression of cyclooxygenase- 2 in the vasculature. Endocrinology 150: 849-861, 2009.

23. Sheppard DC, Rieg G, Chiang LY, Filler SG, Edwards JE Jr and Ibrahim AS: Novel inhalational murine model of invasive pulmonary aspergillosis. Antimicrob Agents Chemother 48: 1908-1911, 2004

24. Joly-Guillou ML, Wolff M, Pocidalo JJ, Walker F and Carbon C: Use of a new mouse model of Acinetobacter baumannii pneumonia to evaluate the postantibiotic effect of imipenem. Antimicrob Agents Chemother 41: 345-351, 1997.
25. McConnell MJ, Hanna PC and Imperiale MJ: Cytokine response and survival of mice immunized with an adenovirus expressing Bacillus anthracis protective antigen domain 4. Infect Immun 74: 1009-1015, 2006

26. Garcia-Quintanilla M, Pulido MR, Pachón J and McConnell MJ: Immunization with lipopolysaccharide-deficient whole cells provides protective immunity in an experimental mouse model of Acinetobacter baumannii infection. PLoS One 9: e114410, 2014.

27. Eveillard M, Soltner C, Kempf M, Saint-André JP, Lemarié C, Randrianarivelo C, Seifert H, Wolff M and Joly-Guillou ML: The virulence variability of different Acinetobacter baumannii strains in experimental pneumonia. J Infect 60: 154-161, 2010.

28. McConnell MJ and Pachón J: Active and passive immunization against Acinetobacter baumannii using an inactivated whole cell vaccine. Vaccine 29: 1-5, 2010.

29. Bentancor LV, O'Malley JM, Bozkurt-Guzel C, Pier GB and Maira-Litrán T: Poly-N-acetyl- $\beta-(1-6)$-glucosamine is a target for protective immunity against Acinetobacter baumannii infections. Infect Immun 80: 651-656, 2012.

30. McConnell MJ, Rumbo C, Bou G and Pachon J: Outer membrane vesicles as an acellular vaccine against Acinetobacter baumannii. Vaccine 29: 5705-5710, 2011.

31. Bentancor LV, Routray A, Bozkurt-Guzel C, Camacho-Peiro A, Pier GB and Maira-Litrán T: Evaluation of the trimeric autotransporter Ata as a vaccine candidate against Acinetobacter baumannii infections. Infect Immun 80: 3381-3388, 2012.

32. Luo G, Lin L, Ibrahim AS, Baquir B, Pantapalangkoor P, Bonomo RA, Doi Y, Adams MD, Russo TA and Spellberg B: Active and passive immunization protects against lethal, extreme drug resistant-Acinetobacter baumannii infection. PLoS One 7: e29446, 2012

33. Russo TA, Beanan JM, Olson R, MacDonald U, Cox AD, St Michael F, Vinogradov EV, Spellberg B, Luke-Marshall NR and Campagnari AA: The K1 capsular polysaccharide from Acinetobacter baumannii is a potential therapeutic target via passive immunization. Infect Immun 81: 915-922, 2013.

34. Lin L, Tan B, Pantapalangkoor P, Ho T, Hujer AM, Taracila MA, Bonomo RA and Spellberg B: Acinetobacter baumannii rOmpA vaccine dose alters immune polarization and immunodominant epitopes. Vaccine 31: 313-318, 2013

35. Fattahian Y, Rasooli I, Mousavi Gargari SL, Rahbar MR, Darvish Alipour Astaneh S and Amani J: Protection against Acinetobacter baumannii infection via its functional deprivation of biofilm associated protein (Bap). Microb Pathog 51: 402-406, 2011.

36. Garcia-Quintanilla M, Pulido MR and McConnell MJ: First steps towards a vaccine against Acinetobacter baumannii. Curr Pharm Biotechnol 14: 897-902, 2013.

37. LaVallie ER, DiBlasio EA, Kovacic S, Grant KL, Schendel PF and McCoy JM: A thioredoxin gene fusion expression system that circumvents inclusion body formation in the E. coli cytoplasm. Biotechnology (N Y) 11: 187-193, 1993. 